\title{
Analysis of prevalence of selected anamnestic factors among women with pelvic organ prolapse
}

\author{
Jakub Śliwa ${ }^{A-F}$, Anna Rosner-Tenerowicz, ${ }^{C, F}$, , Anna Kryza-Ottou ${ }^{B-D, F}$, Sylvester Ottou ${ }^{C, D, F}$,

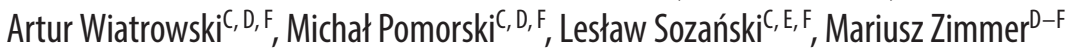 \\ $2^{\text {nd }}$ Clinic of Gynecology and Obstetrics, Wroclaw Medical University, Poland \\ A - research concept and design; B - collection and/or assembly of data; $C$ - data analysis and interpretation; \\ $D$ - writing the article; $E$ - critical revision of the article; $F$ - final approval of the article
}

Address for correspondence

Anna Kryza-Ottou

E-mail: ania.kryza@interia.pl

Funding sources

None declared

Conflict of interest

None declared

Received on May 11, 2016

Reviewed on July 12, 2016

Accepted on February 15, 2017

\section{Abstract}

Background. Pelvic organ prolapse is the most frequent medical condition in women in the postmenopausal age. The pathophysiology is multifactorial.

Objectives. The purpose of this paper was to analyze the prevalence of selected anamnestic factors in the population of women treated due to pelvic organ prolapse in the $2^{\text {nd }}$ Department and Clinic of Obstetrics and Gynecology Wroclaw Medical University (Poland).

Material and methods. A total of 104 medical histories of women treated in the $2^{\text {nd }}$ Department and Clinic of Obstetrics and Gynecology in the years 2012-2013 due to pelvic organ prolapse were analyzed.

Results. The most frequent type of defect was the complex defect concerning both cystocele and rectocele. Intensity of dysfunctions was determined by age, obstetric history (parity, newborn's body mass and process of labor), and woman's constitutional characteristic (her BMl and height). A comparison based on the type of defect revealed no differences between the groups except for BMI, which was the highest in the rectocele group $(31.15 \pm 5.84 ; p=0.0069)$.

Conclusions. The multifactorial ethology and differential clinical presentation including several types of this defect make this disorder difficult to prevent and treat. The obtained results confirm that there exists a relation between the data from the medical history and the prevalence of pelvic organ prolapse. Anamnesis can be useful when predicting prevalence and, in the future, may even help to decrease the prevalence of this type of disorder.

Key words: risk factors, epidemiology, pelvic organ prolapse

DOI

10.17219/acem/68994

Copyright

Copyright by Author(s)

This is an article distributed under the terms of the

Creative Commons Attribution Non-Commercial License

(http://creativecommons.org/licenses/by-nc-nd/4.0/) 


\section{Introduction}

Pelvic organ prolapse is one of the most frequent disorders connected with age that makes women visit their gynecologist. In the United States, this problem may affect even $24 \%$ of the women's population, whereby the percentage depends mainly on age. Among women between 20 and 39 years of age, it concerns $10 \%$ of the population, whereas it involves up to $50 \%$ of women in their eighties. ${ }^{1}$

With regard to the ageing process of the society, this problem will involve a higher rate of the total women's population. One estimates that in 2050 it will concern over 30\% of women over 20 years old. ${ }^{2}$ In the United States, $11.8 \%$ of women have undergone surgical procedures connected with one of the types of prolapse, which constitutes the most common indication for surgical procedure. In developed countries, approx. $20 \%$ of surgical procedures among women are carried out due to pelvic organ prolapse. ${ }^{3-5}$ It is also worth mentioning that the problem is probably more frequent, because only $10 \%$ of the population struggling with pelvic organ prolapse in their everyday life seek help from a gynecologist, and the majority never ask for it. ${ }^{6}$

We can distinguish a few types of pelvic organ prolapse depending on the defect. Anterior prolapse occurs when the supportive tissue between a woman's bladder and vaginal wall weakens and stretches, allowing the bladder to bulge into the vagina (cystocele). Posterior prolapse occurs when the thin wall of fibrous tissue that separates the rectum from the vagina weakens, allowing the vaginal wall to bulge (rectocele). Small bowel prolapse occurs when the small intestine (small bowel) descends into the lower pelvic cavity and pushes at the top part of the vagina, creating a bulge (enterocoele). Uterus and vagina prolapse is with the uterus dislocation toward the vagina opening and finally its prolapse with complete eversion (descensus et prolapsus uteri). The consequences of the prevalence of the disorders are mainly connected with the discomfort they cause in such cases. Among the most frequent symptoms are the following: a sensation of a mass bulging into the vagina, a sensation of something coming or falling out of the vagina, urinary incontinence, fecal incontinence, having to push up on the perineum or digitate the vagina in order to urinate or defecate, discomfort during sexual intercourse. ${ }^{3,7,8}$

A properly functioning pelvic ligamentous and muscular system as well as the pelvic organ support system should sustain pelvic organs in anatomical position, ensuring a comfortable life for every woman regardless of her age. Damage to anatomical structures, connective tissue and nerves leads to different pelvic dysfunctions resulting in pelvic organ prolapse. ${ }^{9-11}$ The reasons for pelvic organ prolapse are complex and arise from mechanical damage to the connective tissue of the true pelvis muscular system, weakening of the connective tissue fibers which follow the incompleteness of tissue structures, and vascularization dysfunctions or innervation of the anatomical structures responsible for sustaining pelvic organs in anatomical position. Among the most common risk factors are perinatal injuries, large body mass of a fetus during delivery, forceps delivery, strenuous physical work, family history of pelvic organ prolapse, obesity, chronic pulmonary disorders, diabetes, and constipation. ${ }^{11-14}$

The objective of this study was to analyze the prevalence of selected anamnestic factors in the population of women treated at the university clinic due to pelvic organ prolapse.

\section{Material and methods}

Medical histories received from 104 patients of the university clinic who were treated due to pelvic organ prolapse in the years 2012-2013 were analyzed, taking into consideration the following factors: frequency and type of defect; education and place of residence; age; smoking habits; constitutional characteristics; parity (number of labors, critical fetus body mass); additional disorders such as diabetes, pulmonary dysfunctions; history of subtotal hysterectomy and total hysterectomy; and history of hormonal replacement therapy (HRT).

The study was conducted in accordance with the Declaration of Helsinki after obtaining approval from the local Bioethics Committee.

Statistical analysis was carried out with the Statistica software package v. 10 (StatSoft Inc., Tulsa, USA). The data was presented as means and percentages. Associations between anamnestic and demographic factors were assessed by Spearman's rank correlation coefficient within the formed groups according to diagnosis. Verification of the hypothesis of means equality for groups of the same diagnosis was performed by one-way analysis of variance (ANOVA test) and Pearson's $\chi^{2}$ test. For posthoc comparison, the method of least significant difference (LSD) was used. The criterion for statistical significance was set at $\mathrm{p}<0.05$.

\section{Results}

The data of 104 patients aged 64.43 years $(S D=9.66)$ was analyzed. The most frequent type of prolapse was the combined defect, which was diagnosed in 49 (47\%) patients. The majority of the study group had increased body weight; only $30 \%$ of patients had their BMI within the normal range. Most of them lived in the city (65\%), and the largest percentage had higher education (46\%). The most frequent type of surgery performed in the patients studied was combined repair (39\%); however, $25 \%$ of patients were treated non-invasively. Half of the study group reported 2 vaginal deliveries in the past. Among chronic diseases, diabetes was the most common and it was reported by $23 \%$ of patients. Most women had not suffered from any gynecological condition in the past, but some reported 
total abdominal hysterectomy (14.29\%), amputation of the body of the uterus (7.79\%), and uterine myoma (9.09\%). It is worth underlining that the standard procedure at our clinic is to suspend the vagina stump or the uterine cervix to round, cruciate and suspensory ligaments in each procedure of subtotal hysterectomy or total hysterectomy. The detailed characteristics of the demographic and clinical data as well as risk factors are presented in Table 1.

Analysis of variance was conducted for subgroups divided according to diagnosis. It did not reveal any differences of means in regard to variables such as age $(p=0.7395)$, age at the last delivery $(\mathrm{p}=0.7378)$, the highest newborn birth weight $(\mathrm{p}=0.9429)$, and patient's height $(\mathrm{p}=0.5134)$. Significant differences were found in regard to patient's body weight $(\mathrm{p}=0.0371)$ and BMI $(\mathrm{p}=0.0069)$. The results of the post-hoc comparison for BMI are shown in Table 1.

Spearman's rank correlation coefficient did not reveal any clinically important correlations between coexisting diseases and the sociodemographic and clinical data from anamnesis.

\section{Discussion}

Our retrospective study on patients treated due to pelvic organ prolapse showed the existence of different risk factors connected with the type and stage of pelvic dysfunction. The most frequent pelvic disorder reported in this group of patients was the defect connected with both cystocele and rectocele. This may lead to the conclusion that cystocele is the most common type of dysfunction throughout the whole group of women with pelvic organ disorders. Similar results were obtained by Hendrix et al. on a large group where the most frequently observed disorder was also cystocele. ${ }^{14}$

Among the women who were examined, the largest percentage of them had higher education $-46 \%$. This stands in contradiction to the data available in the literature, where the largest group included women with primary and vocational education, as in Cooper et al's. paper, in which $45 \%$ of women declared such educational background. ${ }^{6}$ Neither was there a relation between education and the prevalence of pelvic disorders in Chiaffarino et al.'s study, who had examined a representative group of 108 women with moderate or high grade of pelvic organ prolapse. ${ }^{11}$ This contradiction may be the effect of different groups examined in these papers - in the case of Cooper et al.'s, the cross-sectional study concerned a large group of patients with pelvic organ prolapse, while our analysis included patients who were admitted to hospital for pelvic organ prolapse surgery.

The age group and average age of patients who underwent the corrective procedures due to pelvic disorders were defined and compared with the above-mentioned studies. The feedback was similar - the highest proportion of patients belonged to the group of 60-69 years. ${ }^{14}$ Both in
Chiaffarino et al.'s study and Cooper et al.'s study, the age of the patients who underwent procedures due to pelvic organ prolapse in most cases did not exceed 56 years. ${ }^{6,11}$

In numerous reports, no association was found between smoking habits and a higher risk of pelvic disorders. ${ }^{11,14-16}$ Hendrix et al. showed that current smoking is connected with a decrease in the prevalence of both cystocele and rectocele. ${ }^{14}$ In the present study, the highest rate of smokers was noted among the patients with uterine prolapse - 9 patients; however, current smokers and women with smoking habits in their medical history were not diversified.

One of the best documented and frequently discussed prolapse risk factors in the literature is the constitutional status of patients evaluated on the basis of BMI index. Many authors indicate a strong relation between obesity and the prevalence of vaginal wall and uterine descent. $5,14,16,17$ This is explained by increased intraabdominal pressure on the diaphragm along with increased BMI leading to pathological changes such as limitations in the support function and loosening of tissues supporting the pelvic organ in anatomical position. Other authors did not observe any correlation between obesity and the prevalence of pelvic organ prolapse. ${ }^{11}$ Our findings showed significantly higher BMI in the rectocele group; however, our study group included patients of whom $70 \%$ had their BMI above the normal range, confirming the fact that being overweight or obese is associated with the development of prolapse. Due to these discrepancies, many authors attempt to build questionnaires which would make it possible to carry out a classified risk evaluation of the individual prevalence of pelvic organ prolapse based on constitutional data, family history and other data that could help estimate the future risk individually for each patient. An example of such a questionnaire may be the UR-CHOICE system. Here, the woman's height $<160 \mathrm{~cm}$ is said to be the increased risk factor. ${ }^{18,19}$ In our paper, the average height was $160.86 \mathrm{~cm}$; however, it is worth emphasizing that $55.3 \%$ of patients were shorter than $160 \mathrm{~cm}$.

There were not any nulliparae in the examined group, all patients in their medical history declared at least 1 spontaneous delivery, and most were after 2 or more deliveries, which may indicate a strong connection between spontaneous delivery and remote consequences occurring as pelvic organ prolapse. This is in line with the results obtained in earlier population studies. ${ }^{1,11,14,16}$ During spontaneous delivery, support tissues may stretch, muscle fibers may be damaged, and the pelvis fundus structures responsible for sustaining the true pelvis may become partially denervated, which may lead to the dysfunction of the structures, pelvic disorders and urinary incontinence. However, the data from different sources concerning the pathomechanism and possible risks of denervation of the structures of the true pelvis fundus is contradictory. ${ }^{9-11,20,21}$ Recently the role of perinatal damage to the levator ani muscle is being emphasized. The determinants may be age during 
labor, head circumference of the fetus, duration of the $2^{\text {nd }}$ phase of labor, use of forceps, or the fetu's body mass. ${ }^{1}$

In the present study, the average body mass of the biggest of the fetuses born was $3773 \mathrm{~g}$. This can be compared with the result obtained in Erata et al.'s study, where the critical body mass increasing the risk of surgical intervention causing pelvic organ prolapse was $3800 \mathrm{~g} .{ }^{22}$ This study provided information on the relation between the critical body mass and the type of defect, confirming, at the same time, that the occurrence of higher than critical body mass is most frequent among women with more advanced disorders. Chiaffarino et al. did not find a negative influence of the newborn's body mass on subsequent risk of pelvic disorders. ${ }^{11}$ At the same time, Chiaffarino et al.'s study, interestingly, concerned patients who had undergone surgical treatment due to pelvic organ prolapse, which is rather unclear. In our study, the newborn's birth weight was not significantly different among women with the 4 types of pelvic organ prolapse examined; however, the highest newborn's weight appeared in the cystocele and rectocele groups and reached 3850 g. Additionally, $78 \%$ of women with both cystocele and rectocele, and $79 \%$ of women with uterine prolapse had given birth to children of high body mass.

Some studies mention an interesting relation between the prevalence of pelvic organ prolapse and the pregnancy period itself and relaxin secretion among pregnant women. Relaxin is supposed to modulate support tissues and favors the subsequent development of pelvic organ prolapse, which would exclude elective cesarean section as a method of preventing pelvic disorders. ${ }^{5,6,15}$ The above-mentioned conclusions could not be compared with the results obtained in our analysis, because in the examined group of women, none of the patients had had a cesarean section. The matter of the influence of anamnestic factors in this period of pregnancy needs to be analyzed more thoroughly.

Many authors point to pulmonary disorders with chronic cough and diabetes as independent factors increasing the risk of pelvic disorders.,12 In our study, no increased risk was stated concerning the prevalence of these illnesses among patients examined at the $2^{\text {nd }}$ Department and Clinic of Obstetrics and Gynecology in Wrocław, neither was such relation found in Uustal Fornell et al.'s study. ${ }^{22}$

The literature mentions hysterectomy as a factor which has a negative influence on pelvic organs. The pathomechanism of the prolapse is multifactorial and applies to the disorders of few support points according to DeLancey, with the significant role of the tissues alongside the vagina (paracolpium) of the $1^{\text {st }}$ level in situations where proper fixation of the vagina stump apex exists. ${ }^{23,24}$ Our results did not confirm the negative influence of the former procedures, because only $10.6 \%$ of all examined patients had undergone prior abdominal hysterectomy, 5.7\% reported amputation of the body of the uterus, and uterine myoma was observed among $6.7 \%$ of patients. It is worth emphasizing that at our clinic we suspend the vagina stump or uterine cervix to round, cruciate and ovarian suspensory ligaments as a standard procedure in every case when we perform total hysterectomy or subtotal hysterectomy. Most women had not suffered from gynecological disorders in the past. No relation was observed between hysterectomy and the subsequent lowering of the vagina walls and uterine cervix, unless additional suspension of the vagina/uterine cervix was applied during the hysterectomy or amputation of the body of the uterus. An 11-year analysis by Jurića et al. confirms the above-mentioned conclusions. ${ }^{24}$ They applied the technique of suspending the vagina stump during abdominal hysterectomies performed in the group of 982 patients and did not observe posterior stump and vagina wall prolapse.

In our study, no relation was proved regarding the type of defect and applying hormonal substitutive therapy. Similar conclusions may be found in the literature - neither in Chiaffarino et al.'s study nor in Hendrix et al.'s study, is there evidence that using hormonal substitutive therapy is connected with the prevalence of pelvic organ prolapse. ${ }^{11,14}$

In this study, it was shown that the crucial factors relating to the prevalence of pelvic organ prolapse are: parity, method of delivery and critical body mass of the newborn as well as the woman's constitutional characteristics, her BMI and height. These anamnestic factors predominantly determine the prevalence of pelvic organ prolapse and may, in consequence, result in the necessity of applying surgical treatment. The results obtained by our team confirm the connection between the data from medical history and the risk of the prevalence of pelvic organ prolapse. In the future, this might help to predict and perhaps avoid the prevalence of this type of disorder.

This study has its limitations. One of them is the lack of possibility to evaluate more the common ways of delivery as risk factors in the population examined. The reason for this is that the patients who were examined had finished reproduction before the percentage of cesarian sections rose, and none of these women had a cesarian section in their medical history. Another limitation is the fact that the evaluation of prolapse could have been partly subjective, because it was performed based on the Valsava maneuver in speculum and bimanual examination.

\section{Conclusions}

The multifactorial etiology and differential clinical presentation, including several types of this defect, make this disorder difficult to prevent and treat. Our study confirms an association between the data from the medical history and the prevalence of pelvic organ prolapse. Factors such as mode of delivery, newborn's body mass, woman's BMI and height determined the occurrence of pelvic organ prolapse. Patients with different types of pelvic organ prolapse presented similar medical history except for BMI, which was significantly higher in the rectocele group. A therapeutic 


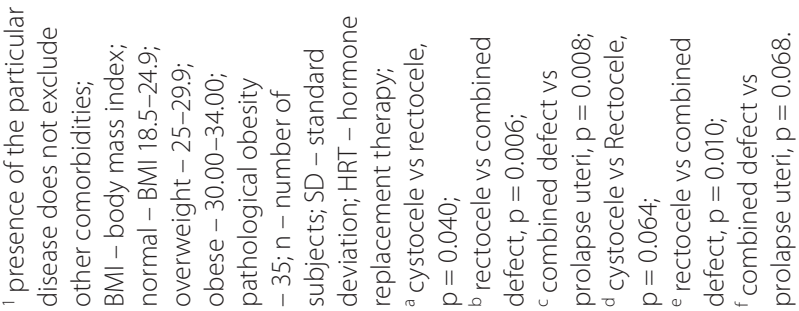




strategy based on the type of pelvic organ prolapse along with the data from anamnesis can be useful in predicting prevalence and, in the future, may even help to decrease the prevalence of this type of disorder.

\section{References}

1. Bozkurt M, Yumru AE, Sahin L. Pelvic floor dysfunction, and effects of pregnancy and mode of delivery on pelvic floor. Taiwan J Obstet Gynecol. 2014;53:452-458.

2. Khan AA, Eilber KS, Clemens JQ, Wu N, Pashos CL, Anger JT. Trends in management of pelvic organ prolapse among female medicare beneficiaries. Am J Obstet Gynecol. 2015;212:463.e1-8.

3. Choi KH, Hong JY. Management of pelvic organ prolapse. Korean J Urol. 2014;55:693-702.

4. Ghetti C, Gregory WT, Clark AL. Risk factors for surgically managed pelvic organ prolapse and urinary incontinence. Int J Gynaecol Obstet. 2007;98:63-64.

5. MacLennan AH, Taylor AW, Wilson DH, Wilson D. The prevalence of pelvic floor disorders and their relationship to gender, age, parity and mode of delivery. BJOG. 2000;107:1460-1470.

6. Cooper J, Annappa M, Dracocardos D, Cooper W, Muller S, Mallen CH. Prevalence of genital prolapsed symptoms in primary care: A crosssectional survey. Int Urogynecol J. 2015;26:505-510.

7. Athanasiou S, Hill S, Gleeson C, Anders K, Cardozo L. Validation of the ICS prolapsed pelvic organ prolapsed descriptive system. Neurourol Urodynam. 1995:14:414-415.

8. Manonai J, Mouritsen L, Palma P, Contreras-Ortiz O, Korte JE, Swift S. The inter-system association between the simplified pelvic organ prolapsed quantification system (S-POP) and the standard pelvic organ prolapsed quantification system (POPQ) in describing pelvic organ prolapsed. Int Urogynecolo J. 2011;22:347-352.

9. Snooks SJ, Swash M, Setchel M, Henry MM. Injury to innervation of pelvic floor sphincter musculature in childbirth. Lancet. 1984;2: 546-550.

10. Snooks SJ, Swash M, Henry MM, Setchel M. Risk factors in childbirth causing damage to the pelvic floor innervation. Int J Colorectal Dis. 1986;1:20-24.

11. Chiaffarino F, Chatenoud L, Dindelli M, et al. Reproductive factors, family history, occupation and risk urogenital prolapse. Eur J Obstet Gynecol Reprod Biol. 1999;82:63-67.
12. Lawrence JM, Lukacz ES, Amy Liu IL, Nager ChW, Luber KM. Pelvic floor disorders, diabetes, and obesity in women: Findings from the Kaiser Permanente Continence Associated Risk Epidemiology Study. Diabetes Care. 2007;30:2536-2541.

13. Ghetti C, Gregory WT, Clark AL. Risk factors for surgically managed pelvic organ prolapse and urinary incontinence. Int J Gynaecol Obstet. 2007;98:63-64.

14. Hendrix SL, Clark A, Nygaard I, Aragaki A, Barnabei V, McTiernan A. Pelvic organ prolapse in the woman's health initiative: Gravity and gravidity Am J Obstet Gynecol. 2002;186:1160-1166.

15. Lonnee-Hoffmann RA, Salvesen O, Morkved S, Schei B. Self-reported pelvic organ prolapse surgery, prevalence, and nonobstetric risk factors: Findings from the Nord Trondelag Health Study. Int Urogynecol J. 2015;26:407-414.

16. Uustal Fornell E, Wingren G, Kjolhede P. Factors associated with pelvic floor dysfunction with emphasis on urinary and fecal incontinence and genital prolapse: An epidemiological study. Acta Obstet Gynecol Scand. 2004;83:383-389.

17. Glazener C, Elders A, MacArthur C, et al. Childbirth and prolapse: Long term associations with the symptoms and objective measurement of pelvic organ prolapse. BJOG. 2013;120:161-168.

18. Wilson D, Dornan J, Milsom I, Freeman R. UR-CHOICE: Can we provide mothers-to-be with information about the risk of future pelvic floor dysfunction. Int Urogybecol J. 2014;25:1449-1152.

19. Giarenis I, Robinson D. Prevention and management of pelvic organ prolapsed. F1000 Prime Reports. 2014;6:77.

20. Smith ARB, Hosker GL, Warrel DW. The role of partial denervation of pelvic floor in the aetiology of genitourinary prolapsed and stress incontinence of urine: A neurophisiological study. Br J Obstet Gynaecol. 1989;96:24-28.

21. Gilpin SA, Gosling JA, Smith ARB, Warrel DW. The pathogenesis of genitourinary prolapsed and stress incontinence of urine: A histological and histochemical study. Br J Obstet Gynaecol. 1989;96:15-23.

22. Erata YE, Kilic B, Guciu S, Saygili U, Uslu T. Risk factors for pelvic surgery. Arch Gynecol Obstet. 2002; 267:14-18.

23. DeLancey JO. Anatomic aspects of vaginal eversion after hysterectomy. Am J Obstet Gynecol. 1992;166(6 Pt 1):1717-1724.

24. Jurić M, Postruznik S, Jurić-Vitanović A. Prevention of vaginal prolapsus after abdominal hysterectomies. Jugos/ Ginekol Opstet. 1981;21: 91-93. [in Yugoslavian]. 